\title{
Associação entre circunferência da cintura e índice de massa corporal de mulheres brasileiras: PNDS 2006
}

\author{
Association between waist circumference and body mass index \\ of Brazilian women: NDHS 2006
}

Fernanda de Oliveira Meller ${ }^{1}$

Carla Ribeiro Ciochetto ${ }^{1}$

Leonardo Pozza dos Santos ${ }^{1}$

Patrícia Abrantes Duval ${ }^{1}$

Maria de Fátima Alves Vieira ${ }^{1}$

Antônio Augusto Schäfer ${ }^{1}$

\footnotetext{
${ }^{1}$ Programa de PósGraduação em Nutrição e Alimentos, Faculdade de Nutrição, Universidade Federal de Pelotas. Campus Universitário Caixa Postal 354. 96.010-900 Pelotas RS Brasil. fe_meller@hotmail.com
}

Abstract The scope of this study was to evaluate the association between waist circumference (WC) and body mass index (BMI) of Brazilian women of childbearing age studied in the most recent National Demographic and Health Survey (NDHS), in 2006. This study is an excerpt of the NDHS, which is a home-based cross-sectional study. The nutritional status of women was assessed by WC and BMI, considering excess weight to be $B M I=25 \mathrm{Kg} / \mathrm{m} 2$ and $W C$ risk to be $=80 \mathrm{~cm}$. To evaluate the statistical significance, Poisson Regression was applied to identify factors associated with WC risk in women with or without excess weight, presenting the p-value corresponding to the Wald test for heterogeneity or linear trend. Of the 14,101 women studied, 45.8\% were overweight and $55.5 \%$ at WC risk. Regarding the association between BMI and WC, it was found that 23.5\% of women who were not overweight showed WC risk. After a stratified analysis by BMI, the prevalence of WC risk among women without excess weight was greatest in the Northeast, $26.0 \%$, and the Southeast, $24.5 \%$, while the South region presented the lowest prevalence at $18.5 \%$. It follows that the anthropometric WC and BMI measures should be used concomitantly since the use of only one of these measures may overlook individuals at risk for various diseases.

Key words Anthropometry, Nutritional status and women's health
Resumo O objetivo do estudo foi analisar a associação entre a circunferência da cintura (CC) e o indice de massa corporal (IMC) de mulheres brasileiras em idade fértil, estudadas na última Pesquisa Nacional de Demografia e Saúde (PNDS), no ano de 2006. Este estudo é um recorte da PNDS, a qual é um estudo seccional, de base domiciliar. $O$ estado nutricional foi avaliado através do IMC e da CC, considerando-se excesso de peso IMC $\geq 25$ $\mathrm{Kg} / \mathrm{m}^{2}$ e CC de risco $\geq 80 \mathrm{~cm}$. Para a avaliação da significância estatística foi usada Regressão de Poisson, que identificou os fatores associados à CC de risco em mulheres com e sem excesso de peso, apresentando-se o valor p correspondente ao teste de Wald para heterogeneidade ou tendência linear. Das 14.101 mulheres estudadas, 45,8\% apresentaram excesso de peso e 55,5\% CC de risco. Em relação à associação entre IMC e CC, constatou-se que 23,5\% das mulheres sem excesso de peso apresentavam CC de risco. Após análise estratificada pelo IMC, a prevalência de CC de risco entre as mulheres sem excesso de peso foi maior nas regiões Nordeste, 26,0\%, e Sudeste, 24,5\%, e menor na região Sul, 18,5\%. Conclui-se que as medidas antropométricas IMC e CC devem ser empregadas concomitantemente, uma vez que a utilização isolada dessas medidas pode desprezar indivíduos com risco para várias patologias.

Palavras-chave Antropometria, Estado nutricional, Saúde da mulher 
Introdução

A obesidade é uma doença multifatorial, causada pelo desequilíbrio entre a quantidade de energia ingerida e o gasto energético total, acarretando um acúmulo de tecido adiposo ${ }^{1}$. Tal doença é considerada um problema de saúde pública tanto em países desenvolvidos quanto naqueles em desenvolvimento, devido ao aumento alarmante de sua prevalência em diversas regiões do mundo ${ }^{1,2}$.

A partir da análise comparativa entre estudos populacionais no Brasil, evidencia-se o aumento das prevalências de excesso de peso e de obesidade na população adulta brasileira. No período de 1974-1975 a 2008-2009, a prevalência de excesso de peso em mulheres aumentou de $28,7 \%$ para $48,0 \%$, enquanto que a de obesidade mais do que duplicou $(8,0 \% \text { para } 16,9 \%)^{3}$.

As medidas mais comuns para avaliar o excesso de peso corporal na prática clínica e em estudos populacionais são o índice de massa corporal (IMC) e a circunferência da cintura $(\mathrm{CC})^{4,5}$. O IMC apresenta uma boa correlação com a gordura corporal ${ }^{6}$ e com as alterações metabólicas associadas à obesidade ${ }^{7,8}$, entretanto, não é uma medida direta de massa gorda e nem sempre reflete com precisão o grau e a distribuição da gordura corporal9. Já a CC tem sido amplamente utilizada na prática clínica como um simples indicador de obesidade central ${ }^{10}$, sendo que, em diversos países, esta medida é um critério diagnóstico para a síndrome metabólica e tem sido incorporada aos guias de prática clínica como tratamento do sobrepeso e obesidade ${ }^{11}$. Segundo Veloso e Silva ${ }^{12}$, o acúmulo de adiposidade na região abdominal é fator de risco para doenças endócrinas, metabólicas e cardiovasculares, mesmo quando o IMC está dentro dos limites da normalidade.

Embora evidências sugiram que seja utilizada a CC ao invés do IMC para explicar os riscos à saúde relacionados à obesidade ${ }^{13}$, o uso do IMC não deve ser negligenciado. Estes indicadores associados contribuem de forma independente para predizer gordura não abdominal, subcutânea abdominal e visceral ${ }^{14}$

Em geral, há uma falta de coerência na escolha e na utilização de indicadores antropométricos em estudos populacionais ${ }^{15}$ Dessa forma, o objetivo do presente estudo foi analisar a associação entre a CC e o IMC de mulheres brasileiras em idade fértil estudadas na Pesquisa Nacional de Demografia e Saúde no ano de 2006 (PNDS 2006).

\section{Metodologia}

O presente estudo compreende um recorte da PNDS - $2006^{16}$, que se trata de um estudo seccional, de âmbito nacional e de base domiciliar. A pesquisa foi realizada entre os anos de 2006 e 2007, com o objetivo de caracterizar a população feminina brasileira em idade fértil ( 15 a 49 anos de idade) e as crianças menores de cinco anos de idade.

O plano amostral da PNDS $-2006{ }^{16}$ foi desenhado para fornecer estimativas representativas das mulheres brasileiras em idade fértil residentes em domicílios particulares em setores comuns ou não especiais (inclusive favelas), selecionados em dez estratos amostrais que compõem uma combinação de todas as cinco grandes regiões geográficas brasileiras e as áreas urbanas e rurais.

O estudo foi realizado por meio de uma amostra probabilística de domicílios obtida em dois estágios de seleção: as unidades primárias, que são os setores censitários, e as unidades secundárias, que são os domicílios particulares, ocupados ou não ocupados.

Ao final do trabalho de campo foram obtidas informações sobre 14.617 domicílios, nos quais 13.056 residiam pelo menos uma mulher elegível, totalizando 15.575 mulheres entrevistadas.

As informações da pesquisa foram coletadas em dois questionários que continham informações básicas sobre o domicílio e seus moradores habituais e informações detalhadas sobre o público-alvo, com relação a temas, como: reprodução, história de nascimentos, gravidezes, sexualidade, nutrição e medicamentos. A todos os domić́lios selecionados na amostra foi aplicado o questionário Ficha do Domicílio, preferencialmente ao responsável e, na ausência deste, a um morador maior de quinze anos de idade que soubesse fornecer informações sobre o domicílio e seus moradores. Após, foi aplicado o Questionário da Mulher a todas as mulheres elegíveis identificadas como moradoras habituais do domicílio.

No caso de domicílio sorteado sem a presença de um elegível, foi aplicada somente a Ficha do Domicílio até a pergunta sobre a educação do responsável pelo domicílio. Este domicílio foi, então, substituído pelo domicílio elegível mais próximo na sequência do arrolamento, a fim de se obter um total de doze domicílios dentro de um mesmo setor.

Após a aplicação dos questionários, foi mensurado o peso, a estatura e a CC das mulheres de acordo com as recomendações da Organização Mundial da Saúde (OMS) ${ }^{17}$. Essas medidas fo- 
ram feitas duas vezes para cada pessoa, calculando-se a média aritmética de ambas. A estatura foi mensurada em aparelho denominado estadiômetro, com extensão de $210 \mathrm{~cm}$ e precisão de $0,1 \mathrm{~cm}$. A medida do peso foi obtida em balança eletrônica portátil da marca Dayhome ${ }^{\circledR}$, com capacidade de $150 \mathrm{Kg}$ e precisão de $0,1 \mathrm{Kg}$. A mensuração da CC foi realizada no ponto médio entre a última costela e a crista ilíaca e, para esta medida, foi utilizada fita inextensível com capacidade de $150 \mathrm{~cm}$ e precisão de $0,1 \mathrm{~cm}$. Essas medidas antropométricas foram coletadas por antropometristas treinados e todos os equipamentos foram calibrados no início e no final de cada dia de trabalho.

Para todas as pessoas entrevistadas, foi lido o termo de consentimento livre e esclarecido, o qual contemplava todas as informações sobre a pesquisa e assinado por aquelas que aceitaram participar.

O programa utilizado para a entrada de dados foi o CSPro (Census and Survey Processing System), software desenvolvido pelo Bureau do Censo Norte-Americano. O relatório final e a metodologia detalhada da PNDS - 2006 estão publicamente disponibilizados ${ }^{16}$.

No presente estudo, para avaliar o estado nutricional, foi utilizado o IMC e a CC conforme recomendações da $\mathrm{OMS}^{17}$. Em relação ao IMC, os valores inferiores a $16 \mathrm{Kg} / \mathrm{m}^{2}$ foram excluí$\operatorname{dos}^{17}$ e, após, foram consideradas sem excesso de peso aquelas que apresentaram valores inferiores a $25 \mathrm{Kg} / \mathrm{m}^{2}$ e com excesso de peso as com valores iguais ou superiores a $25 \mathrm{Kg} / \mathrm{m}^{2}$. Quanto à CC, as mulheres adultas que apresentaram valores inferiores a $80 \mathrm{~cm}$ foram classificadas como sem risco à saúde e aquelas com valores iguais ou superiores a $80 \mathrm{~cm}$, classificadas como apresentando risco. Em função de não se dispor de valores críticos aceitos internacionalmente para a CC em adolescentes, este indicador foi utilizado apenas para as mulheres com faixa etária de 18 anos ou mais, com isso, a amostra estudada constitui-se de 14.101.

A prevalência da CC foi observada segundo o IMC e as seguintes variáveis de exposição: macrorregião brasileira, situação de domicílio (urbano ou rural), escolaridade (anos completos), cor da pele (referida pela entrevistada), idade (anos), situação conjugal, trabalho fora do lar e número de partos. Para a avaliação da significância estatística, foi utilizada Regressão de Poisson para identificar os fatores associados à CC de risco em mulheres com e sem excesso de peso, apresentando-se o valor $\mathrm{p}$ correspondente ao teste de Wald para heterogeneidade ou tendência linear $(\mathrm{p}<0,05)$.

Os dados foram analisados no programa estatístico Stata (versão 11.0), utilizando o comando svyset para definir os pesos amostrais e conglomerados e o prefixo svy em todas as análises realizadas, tendo em vista a complexidade do processo de amostragem.

O presente estudo foi aprovado pelo Comitê de Ética em Pesquisa da Faculdade de Medicina da Universidade Federal de Pelotas.

\section{Resultados}

A partir dos 13.056 domicílios com pelo menos uma mulher elegível, foi identificado um total de 15.575 mulheres. Destas, 14.101 tinham 18 anos de idade ou mais.

$\mathrm{Na}$ Tabela 1 estão apresentadas as características socioeconômica, demográficas e antropométricas da amostra estudada. Observa-se que a maioria das mulheres residia na região Sudeste $(44,5 \%)$ e na zona urbana do país $(83,9 \%)$, referiu ter cor da pele não branca $(59,2 \%)$ e cerca de $66,0 \%$ referiu ter tido um ou dois partos. Em relação ao IMC, aproximadamente metade das mulheres apresentou excesso de peso $(45,8 \%)$. Quanto à CC, mais da metade da amostra $(55,5 \%)$ apresentou risco.

A Figura 1 mostra a associação entre IMC e CC. Evidencia-se que 23,5\% das mulheres sem excesso de peso apresentaram valores de $\mathrm{CC} \geq$ $80 \mathrm{~cm}(\mathrm{p}<0,001)$.

Após análise estratificada pelo IMC, constatou-se que a prevalência de CC de risco entre as mulheres sem excesso de peso foi maior nas regiões Nordeste, 26,0\%, e Sudeste, 24,5\%, e menor na região Sul, 18,5\%. Entre as mulheres sem excesso de peso, observou-se uma associação inversa, no limiar da significância, entre idade e CC de risco, ou seja, conforme aumenta a idade diminui a prevalência de mulheres com CC de risco. Em relação ao número de partos, as maiores prevalências de CC de risco foram encontradas entre aquelas mulheres sem excesso de peso que referiram um parto e naquelas com excesso de peso e nenhum parto (Tabela 2).

\section{Discussão}

Dentre os aspectos positivos do presente estudo está o fato da amostra estudada ser representativa das mulheres brasileiras de 18 a 49 anos, ten- 
Tabela 1. Características socioeconômica, demográficas e antropométricas de mulheres brasileiras de 18 a 49 anos. PNDS, 2006. $(\mathrm{n}=14.101)$.

\begin{tabular}{|c|c|c|}
\hline Variáveis & $\mathbf{n}$ & $\%$ \\
\hline \multicolumn{3}{|l|}{ Macrorregião } \\
\hline Norte & 2.325 & 7,3 \\
\hline Nordeste & 2.836 & 25,9 \\
\hline Sudeste & 3.055 & 44,5 \\
\hline Sul & 3.026 & 15,0 \\
\hline Centro-oeste & 2.859 & 7,3 \\
\hline \multicolumn{3}{|l|}{ Situação de domicílio } \\
\hline Urbano & 10.047 & 83,9 \\
\hline Rural & 4.054 & 16,1 \\
\hline \multicolumn{3}{|c|}{ Escolaridade (anos completos) } \\
\hline $0-4$ & 6.119 & 48,7 \\
\hline $5-8$ & 2.999 & 23,7 \\
\hline $9-11$ & 2.988 & 24,2 \\
\hline 12 ou mais & 485 & 3,4 \\
\hline \multicolumn{3}{|l|}{ Cor da pele } \\
\hline Branca & 5.475 & 40,8 \\
\hline Não branca & 8.494 & 59,2 \\
\hline \multicolumn{3}{|l|}{ Idade (anos) } \\
\hline $18-24$ & 3.522 & 26,0 \\
\hline $25-32$ & 3.817 & 26,0 \\
\hline $33-40$ & 3.421 & 24,0 \\
\hline $41-49$ & 3.341 & 24,0 \\
\hline \multicolumn{3}{|l|}{ Situação conjugal } \\
\hline Com companheiro & 9.786 & 68,7 \\
\hline Sem companheiro & 4.302 & 31,3 \\
\hline \multicolumn{3}{|l|}{ Trabalho fora do lar } \\
\hline $\operatorname{Sim}$ & 6.978 & 51,2 \\
\hline Não & 7.120 & 48,8 \\
\hline \multicolumn{3}{|l|}{ Número de partos ${ }^{\mathrm{a}}$} \\
\hline Nenhum & 215 & 2,4 \\
\hline 1 & 2.961 & 32,3 \\
\hline 2 & 3.570 & 34,1 \\
\hline 3 ou mais & 4.226 & 31,2 \\
\hline \multicolumn{3}{|l|}{ Índice de Massa Corporal } \\
\hline Sem excesso de peso & 7.420 & 54,2 \\
\hline Com excesso de peso ${ }^{\mathrm{b}}$ & 6.299 & 45,8 \\
\hline \multicolumn{3}{|l|}{ Circunferência da cintura } \\
\hline Sem risco & 5.921 & 44,5 \\
\hline $\mathrm{Com} \mathrm{risco}^{c}$ & 7.386 & 55,5 \\
\hline
\end{tabular}

a Percentual máximo de observações desconhecidas: 22,2\% $(\mathrm{n}=3129)$. ${ }^{\mathrm{b}}$ Índice de massa corporal $\geq 25 \mathrm{Kg} / \mathrm{m}^{2}$.

${ }^{c}$ Circunferência da cintura $\geq 80 \mathrm{~cm}$.

do em vista o processo amostral utilizado. Além disso, a supervisão do trabalho de campo juntamente com a padronização da coleta de medidas antropométricas empregada pela equipe da PNDS - 2006 são outros aspectos importantes a serem destacados.

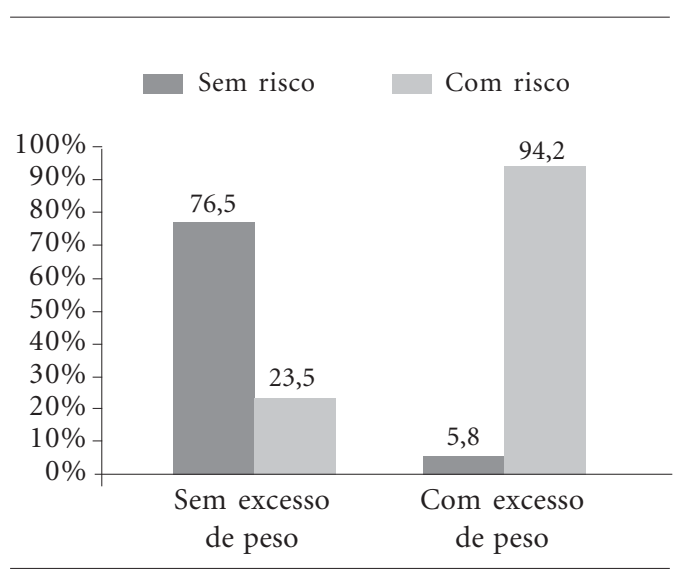

Figura 1. Associação entre a circunferência da cintura e o índice de massa corporal de mulheres brasileiras de 18 a 49 anos. PNDS, 2006. $(\mathrm{n}=14.101)$.

$\mathrm{p}<0,001$ teste de Wald para heterogeneidade.

No presente estudo, foram observadas prevalências de $45,8 \%$ de excesso de peso e $55,5 \%$ de $\mathrm{CC}$ de risco. No que diz respeito à associação entre IMC e CC, evidenciou-se, como era esperado, que a ampla maioria das mulheres com excesso de peso, segundo o IMC, apresentou CC elevada. Contudo, é importante salientar que cerca de 1/4 das mulheres sem excesso de peso apresentaram CC de risco. Essa prevalência foi superior àquelas encontradas em estudos nacional e internacional ${ }^{12,18}$. Em um estudo realizado no estado do Maranhão, os autores encontraram uma prevalência de $15,5 \%$ de obesidade abdominal em mulheres eutróficas ${ }^{12}$, utilizando pontos de corte para classificar o estado nutricional das mulheres iguais aos do presente estudo. Contudo, SongMink et al. ${ }^{18}$, em estudo de base populacional realizado na China, constataram que $8,1 \%$ das mulheres eutróficas (IMC $<24 \mathrm{Kg} / \mathrm{m}^{2}$ ) e $61,2 \%$ das com sobrepeso (IMC entre 24 e $27,9 \mathrm{Kg} / \mathrm{m}^{2}$ ) apresentaram obesidade abdominal. Cabe ressaltar que os pontos de corte utilizados para definir sobrepeso e obesidade abdominal foram diferentes daqueles usados no presente estudo, $\mathrm{o}$ que pode, em parte, explicar a discrepância dos resultados. Lahti-koski et al. ${ }^{15}$ em um estudo de base populacional conduzido na Finlândia, por um período de 15 anos, observaram aumento de $4,3 \mathrm{~cm}$ na média da CC de mulheres, sem que houvesse alteração notável nos valores de IMC.

No que diz respeito à macrorregião de moradia, observou-se uma diferença entre as prevalências de CC elevada em mulheres sem excesso de peso de acordo com as regiões do país, sendo mai- 
Tabela 2. Fatores associados à circunferência da cintura de risco $(\geq 80 \mathrm{~cm})$ em mulheres brasileiras de 18 a 49 anos com e sem excesso de peso. PNDS, 2006. $(n=7.212)$.

\begin{tabular}{|c|c|c|c|c|c|}
\hline \multirow[b]{2}{*}{ Variáveis } & \multicolumn{2}{|c|}{ Sem excesso de peso } & \multicolumn{2}{|c|}{ Com excesso de peso } & \multirow[b]{2}{*}{ Valor $\mathbf{p}$} \\
\hline & $\mathbf{n}$ & $\%$ & $\mathbf{n}$ & $\%$ & \\
\hline Macrorregião & & & & & $<0,001^{\mathrm{a}}$ \\
\hline Norte & 301 & 23,4 & 884 & 76,6 & \\
\hline Nordeste & 386 & 26,0 & 1.093 & 74,0 & \\
\hline Sudeste & 398 & 24,5 & 1.220 & 75,5 & \\
\hline Sul & 281 & 18,5 & 1.223 & 81,5 & \\
\hline Centro-oeste & 295 & 19,5 & 1.131 & 80,5 & \\
\hline Situação de domicílio & & & & & $0,696^{\mathrm{a}}$ \\
\hline Urbano & 1.204 & 23,7 & 3.853 & 76,3 & \\
\hline Rural & 456 & 23,0 & 1.698 & 77,0 & \\
\hline Escolaridade (anos completos) & & & & & $0,774^{\mathrm{b}}$ \\
\hline $0-4$ & 706 & 23,9 & 2.413 & 76,1 & \\
\hline $5-8$ & 359 & 22,2 & 1.140 & 77,8 & \\
\hline $9-11$ & 367 & 24,0 & 1.217 & 76,0 & \\
\hline 12 ou mais & 64 & 21,2 & 184 & 78,8 & \\
\hline Cor da pele & & & & & $0,382^{\mathrm{a}}$ \\
\hline Branca & 643 & 24,6 & 2.112 & 75,4 & \\
\hline Não branca & 1.013 & 23,1 & 3.386 & 76,9 & \\
\hline Idade (anos) & & & & & $0,056^{\mathrm{b}}$ \\
\hline $18-24$ & 287 & 25,8 & 713 & 74,2 & \\
\hline $25-32$ & 445 & 24,5 & 1.328 & 75,5 & \\
\hline $33-40$ & 489 & 24,7 & 1.606 & 75,3 & \\
\hline $41-49$ & 440 & 20,9 & 1.904 & 79,1 & \\
\hline Situação conjugal & & & & & $0,080^{\mathrm{a}}$ \\
\hline Com companheiro & 1.232 & 22,6 & 4.309 & 77,4 & \\
\hline Sem companheiro & 426 & 26,5 & 1.239 & 73,5 & \\
\hline Trabalho fora do lar & & & & & $0,398^{\mathrm{a}}$ \\
\hline Sim & 867 & 24,2 & 2.782 & 75,8 & \\
\hline Não & 794 & 22,8 & 2.768 & 77,2 & \\
\hline Número de partos & & & & & $0,010^{\mathrm{a}}$ \\
\hline Nenhum & 20 & 15,4 & 69 & 84,6 & \\
\hline 1 & 369 & 27,6 & 1.012 & 72,4 & \\
\hline 2 & 460 & 21,4 & 1.628 & 78,6 & \\
\hline 3 ou mais & 566 & 20,5 & 2.208 & 79,5 & \\
\hline
\end{tabular}

${ }^{a}$ teste de Wald para heterogeneidade. ${ }^{b}$ teste de Wald para tendência linear.

or na região Nordeste. Não há pesquisas com amostra representativa da população brasileira que tenham apresentado resultados semelhantes ao do presente estudo, comparando tal achado entre as macrorregiões. No entanto, há estudos locais que observaram esse tipo de associação. Em um realizado na cidade de Pelotas, RS, os autores evidenciaram que cerca de 25\% das mulheres eutróficas apresentaram excesso de gordura abdominal ${ }^{19}$. Já, um realizado na região Nordeste apontou que menos de $1 / 5$ das mulheres classificadas como normais segundo o IMC apresentaram CC de risco ${ }^{12}$.

Observou-se, no presente estudo, associação no limiar da significância entre idade e CC de risco, ou seja, conforme aumenta a idade há uma diminuição da prevalência da CC de risco entre as mulheres sem excesso de peso. Tal resultado não está em consonância com o que diz a literatura, visto que alguns estudos ${ }^{20-23}$ mostram uma relação direta entre as medidas de IMC e CC com a idade. Em um trabalho conduzido por Oka et al. ${ }^{20}$, estudando mulheres japonesas com faixa etária entre 38 e 60 anos, verificou-se que o aumento da idade foi fortemente relacionado à obesidade abdominal. Estudo realizado na cidade de Salvador, BA, evidenciou que tanto o IMC quanto a CC elevados foram associados ao aumento da idade ${ }^{21}$. Ademais, estudo no Rio de Janeiro, RJ, verificou que mulheres com idade superior a 35 anos apresentaram maior prevalência de obesi- 
dade abdominal ${ }^{22}$. A diferença entre os resultados encontrados nesses trabalhos e o do presente estudo pode ser, parcialmente, explicada pelo fato de que tais estudos não realizaram análise estratificada pelo IMC; além disso, utilizaram amostras específicas de determinados locais.

Os resultados do presente estudo evidenciaram associação entre CC de risco e número de partos entre as mulheres com e sem excesso de peso. As maiores prevalências de CC de risco foram observadas nas mulheres nulíparas e com excesso de peso $(84,6 \%)$ e naquelas primíparas sem excesso de peso (27,6\%). Em contrapartida, estudo na cidade do Rio de Janeiro, RJ, evidenciou maior prevalência de CC de risco em mulheres com dois ou mais filhos. Porém, a partir dos modelos de regressão logística, os autores verificaram que quando a população foi estratificada em com ou sem sobrepeso, a associação desapareceu para a CC $>80 \mathrm{~cm}^{22}$.

Estudo de revisão realizado por Seidell ${ }^{24}$ evidenciou que a obesidade abdominal avaliada pela CC está relacionada a um risco maior de mortalidade, em todas as faixas de IMC, mas principalmente em adultos jovens e com IMC relativamente baixo. Tais resultados enfatizam a importância de se incluir a medida da CC como componente básico da avaliação do estado nutricional na prática clínica e em estudos epidemiológi- cos, já que as medidas de CC juntamente ao IMC fornecem informações importantes sobre o risco de doenças ${ }^{25}$.

Algumas limitações precisam ser consideradas, como diferenças entre os métodos para avaliar, definir e categorizar o estado nutricional de mulheres, o que prejudica a comparação com outros estudos que associam IMC e CC. Além disso, a ausência de valores críticos aceitos internacionalmente para a CC em adolescentes impossibilitou a análise para mulheres com idade inferior a 18 anos.

Diante do exposto, pode-se concluir que as medidas antropométricas IMC e CC devem ser empregadas concomitantemente, uma vez que sua utilização isolada pode não detectar indivíduos com risco para diversas patologias associadas a níveis elevados de adiposidade, tais como diabetes mellitus tipo 2, hipertensão arterial sistêmica e dislipidemias ${ }^{26}$, como mulheres sem excesso de peso, mas que apresentam CC de risco. Além disso, devido à elevada prevalência de mulheres com IMC e CC elevados, fazem-se necessárias políticas públicas de saúde que visem não somente a prevenção e o tratamento do excesso de peso, mas também da obesidade abdominal, a fim de promover o estado nutricional adequado das mulheres brasileiras e reduzir as prevalências das doenças associadas.

\section{Colaboradores}

FO Meller foi responsável pela revisão de literatura, análise dos dados, confecção das tabelas, interpretação e redação do artigo final. AA Schäfer, CR Ciochetto, LP Santos, PA Duval e MFA Vieira foram responsáveis pela confecção das tabelas, interpretação e redação do artigo final. 


\section{Referências}

1. Martorell R. Obesity in the developing world. In Caballero B, Popkin BM, editors. The nutrition tran sition: diet and disease in the developing world. London: Academic Press; 2002. p. 147-164.

2. Martorell R, Khan LK, Hughes ML, GrummerStrawn LM. Obesity in women from developing countries. Eur J Clin Nutr 2000; 54(3):247-252.

3. Instituto Brasileiro de Geografia e Estatística (IBGE). Pesquisa de Orçamentos Familiares 2008-2009. Antropometria e Estado Nutricional de Crianças, Adolescentes e Adultos no Brasil. Rio de Janeiro: IBGE; 2010.

4. Gallagher D, Visser M, Sepúlveda D, Pierson RN, Harris T, Heymsfield SB. How useful is body mass index for comparison of body fatness across age, sex, and ethnic Groups? Am J Epid 1999; 143(3):228239.

5. Angelo P, Faith MS, Allison DB, Gallagher D, Chiumello G, Heymsfield SB. Body mass index as a measure of adiposity among children and adolescents: a validation study. J Pediatrics 1998; 132(2): 204-210.

6. Anjos LA. Diagnóstico de obesidade e determinação de requerimentos nutricionais: desafios para a área de Nutrição. Cien Saude Colet 2013; 18(2):294294.

7. Martins IS, Marinho SP. O potencial diagnóstico dos indicadores da obesidade centralizada. Rev Saude Publica 2003; 37(6):760-767.

8. Souza LJ, Neto CG, Chalita FEB, Reis AFF, Bastos DA, Souto Filho JTD, Souza TF, Côrtes VA. Prevalência de obesidade e fatores de risco cardiovasculares em Campos, Rio de Janeiro. Arq Bras Endocrinol Metab 2003; 47(6):669-676.

9. Hsieh SD, Yoshinaga H, Muto T. Waist-to-height ratio, a simple and practical index for assessing central fat distribution and metabolic risk in Japanese men and women. Int J Obes 2003; 27(5):610-616.

10. National Institutes of Health. Clinical guidelines on the identification, evaluation, and treatment of overweight and obesity in adults - the evidence report. Obes Res 1998; 6(Supl. 2):51-209.

11. Mason C, Katzmarzyk PT. Variability in waist circumference measurements according to anatomic measurement site. Obesity 2009; 17(9):1789-1795.

12. Veloso HJF, Silva AAM. Prevalência e fatores associados à obesidade abdominal e ao excesso de peso em adultos maranhenses. Rev Bras Epidemiol 2010; 13(3):400-412.

13. Janssen I, Katzmarzyk PT, Ross R. Waist circumference and not body mass index explains obesity-related health risk. Am J Clin Nutr 2004; 79(3):379-384. Janssen I, Heymsfield SB, Allison DB, Kotler DP,

14. Ross R. Body mass index and waist circumference independently contribute to the prediction of nonabdominal, abdominal subcutaneous, and visceral fat. Am J Clin Nutr 2002; 75(4):683-688.

15. Lahti-koski M, Harald K, Männistö S, Laatikainen $\mathrm{T}$, Jousilahti P. Fifteen-year changes in body mass index and waist circumference in Finnish adults. Eur J Cardiovasc Prev Rehabil 2007; 14(3):398-404.
16. Brasil. Ministério da Saúde (MS). Relatório Final da Pesquisa Nacional de Demografia e Saúde. Pesquisa Nacional de Demografia e Saúde da Criança e da Mulher. Brasília: MS; 2006. [página na Internet]. [acessado 2013 out 13]. Disponível em: http://www. saude.gov.br/pnds2006

17. World Health Organization (WHO). Physical Status: the Use and Interpretation of Anthropometry. Report of a WHO Expert Committee. Geneva: WHO; 1995. (WHO Technical Report Series, 854)

18. Song-Ming Du, Guan-Sheng Ma, Yan-Ping Li, HongYun Fang, Xiao-Qi Hu, Xiao-Guang Yang, Hu YH. Relationship of Body Mass Index, Waist Circumference and Cardiovascular Risk Factors in Chinese Adult. Biomed Environ Sci 2010; 23(2):92-101.

19. Olinto MTA, Nácul LC, Dias-da-Costa JS, Gigante DP, Menezes AMB, Macedo S. Níveis de intervenção para obesidade abdominal: prevalência e fatores associados. Cad Saude Publica 2006; 22(6):12071215.

20. Oka R, Miura K, Sakurai M, Nakamura K, Yagi K, Miyamoto S, Moriuchi T, Mabuchi H, Yamagishi M, Takeda Y, Hifumi S, Inazu A, Nohara A, Kawashiri MA, Kobayashi J. Comparison of waist circumference with body mass index for predicting abdominal adipose tissue. Diabetes Res and Clin Pract 2009; 83(1):100-105.

21. Oliveira LPM, Assis AMO, Silva MCM, Santana MLP, Santos NS, Pinheiro SMC, Barreto ML, Souza CO. Fatores associados a excesso de peso e concentracão de gordura abdominal em adultos na cidade de Salvador, Bahia, Brasil. Cad Saude Publica 2009; 25(3):570-582.

22. Kac G, Velásquez-Meléndez G, Coelho MASC. Fatores associados à obesidade abdominal em mulheres em idade reprodutiva. Rev Saude Publica 2001; 35(1):46-51.

23. Correia LL, Silveira DMI, Silva AC, Campos JS, Machado MMT, Rocha HAL, Cunha AJLA, Lindsay AC. Prevalência e determinantes de obesidade e sobrepeso em mulheres em idade reprodutiva residentes na região semiárida do Brasil. Cien Saude Colet 2011; 16(1):133-145.

24. Seidell JC. Waist circumference and waist/hip ratio in relation to all-cause mortality, cancer and sleep apnea. Eur J Clin Nutr 2010; 64(1):35-41.

25. Zhu S, Heshka S, Wang Z, Shen W, Alisson DB, Ross R, Heymsfield SB. Combination of BMI and waist circumference for identifying cardiovascular risk factors in whites. Obes Res 2004; 12(4):633-645.

26. Aekplakorn W, Kosulwat V, Suriyawongpaisal P. Obesity indices and cardiovascular risk factors in Thai adults. Int J Obes 2006; 30(12):1782-1790.

Artigo apresentado em 05/08/2012

Aprovado em 17/10/2012

Versão final apresentada em 30/10/2012 
